PROCEEDINGS OF THE

AMERICAN MATHEMATICAL SOCIETY

Volume 138, Number 12, December 2010, Pages 4311-4320

S 0002-9939(2010)10421-5

Article electronically published on May 20, 2010

\title{
TREE METRICS AND THEIR LIPSCHITZ-FREE SPACES
}

\author{
A. GODARD
}

(Communicated by Nigel J. Kalton)

\begin{abstract}
We compute the Lipschitz-free spaces of subsets of the real line and characterize subsets of metric trees by the fact that their Lipschitz-free space is isometric to a subspace of $L_{1}$.
\end{abstract}

\section{INTRODUCTION}

Let $M$ be a pointed metric space (a metric space with a designated origin denoted $0)$. We denote by $\operatorname{Lip}_{0}(M)$ the space of all real-valued Lipschitz functions on $M$ which vanish at 0 equipped with the standard Lipschitz norm

$$
\|f\|=\inf \{K \in \mathbb{R} / \forall x, y \in M|f(y)-f(x)| \leq K d(x, y)\} .
$$

The closed unit ball of this space being compact for the topology of pointwise convergence on $M, \operatorname{Lip}_{0}(M)$ has a canonical predual, namely the closed linear span of the evaluation functionals $\delta_{x}$ (whether this predual is unique up to a linear isometry is an open question). We call Lipschitz-free space over $M$ and denote by $\mathcal{F}(M)$ this predual. Lipschitz-free spaces are studied extensively in [12] where they are called Arens-Eells spaces. Note that for every point $a$ of $M, f \mapsto f-f(a)$ is a weak*-weak* continuous linear isometry between $\operatorname{Lip}_{0}(M)$ and $\operatorname{Lip}_{a}(M)$ : the choice of different base points therefore yields isometric Lipschitz-free spaces.

The key property of the Lipschitz-free space $\mathcal{F}(M)$ is that a Lipschitz map between two metric spaces admits a linearization between the corresponding Lipschitzfree spaces. More precisely, if $M_{1}$ and $M_{2}$ are pointed metric spaces and $L$ : $M_{1} \rightarrow M_{2}$ is a Lipschitz map satisfying $L(0)=0$, there exists a unique linear map $\widehat{L}: \mathcal{F}\left(M_{1}\right) \rightarrow \mathcal{F}\left(M_{2}\right)$ such that the following diagram commutes:

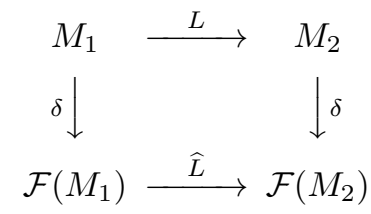

(we refer to 6] for details on this functorial property). In particular, $\mathcal{F}\left(M^{\prime}\right)$ is an isometric subspace of $\mathcal{F}(M)$ whenever $M^{\prime}$ isometrically embeds into $M$.

Received by the editors May 11, 2009 and, in revised form, January 29, 2010.

2010 Mathematics Subject Classification. Primary 46B04; Secondary 05C05, 46B25, 54E35.

Key words and phrases. Lipschitz-free spaces, subspaces of $L_{1}$, metric trees, four-point property. 
Differentiation almost everywhere yields a weak*-weak* continuous linear isometry between $\operatorname{Lip}_{0}(\mathbb{R})$ and $L_{\infty}$ which predualizes into a linear isometry between $\mathcal{F}(\mathbb{R})$ and $L_{1}$ (the discrete version of this argument provides $\mathcal{F}(\mathbb{N}) \equiv \ell_{1}$ ).

According to a result of Godefroy and Talagrand ([7]), any Banach space isomorphic to a subspace of $L_{1}$ is a unique isometric predual of its dual: given the open question mentioned above, it is therefore natural to highlight metric spaces which admit a subspace of $L_{1}$ as their Lipschitz-free space.

The purpose of this article is to show that $\mathcal{F}(M)$ is linearly isometric to a subspace of $L_{1}$ if and only if $M$ isometrically embeds into an $\mathbb{R}$-tree (i.e. a metric space where any couple of points is connected by a unique arc isometric to a compact interval of the real line). This gives a complement to a result of Naor and Schechtman that $\mathcal{F}\left(\mathbb{R}^{2}\right)$ is not isomorphic to a subspace of $L_{1}([10)$.

We also compute the Lipschitz-free spaces of subsets of the real line by integrating bounded measurable functions with respect to a measure adapted to the subset considered.

\section{Preliminaries}

In this section we define differentiation for functions defined on a subset of a pointed $\mathbb{R}$-tree and introduce measures which enable us to retrieve the values of such absolutely continuous functions by integrating their derivative.

We first recall the definition of $\mathbb{R}$-trees and define the analogues of the Lebesgue measure for those metric spaces (this measure is called the length measure).

Definition 2.1. An $\mathbb{R}$-tree is a metric space $T$ satisfying the following two conditions.

(1) For any points $a$ and $b$ in $T$, there exists a unique isometry $\phi$ of the closed interval $[0, d(a, b)]$ into $T$ such that $\phi(0)=a$ and $\phi(d(a, b))=b$.

(2) Any one-to-one continuous mapping $\varphi:[0,1] \rightarrow T$ has the same range as the isometry $\phi$ associated to the points $a=\varphi(0)$ and $b=\varphi(1)$.

If $T$ is an $\mathbb{R}$-tree, we denote for any $x$ and $y$ in $T, \phi_{x y}$ the unique isometry associated to $x$ and $y$ as in definition 2.1 and write $[x, y]$ for the range of $\phi_{x y}$; such subsets of $T$ are called segments. We say that a subset $A$ of $T$ is measurable whenever $\phi_{x y}^{-1}(A)$ is Lebesgue-measurable for any $x$ and $y$ in $T$. If $A$ is measurable and $S$ is a segment $[x, y]$, we write $\lambda_{S}(A)$ for $\lambda\left(\phi_{x y}^{-1}(A)\right)$ where $\lambda$ is the Lebesgue measure on $\mathbb{R}$. We denote by $\mathcal{R}$ the set of all subsets of $T$ which can be written as a finite union of disjoint segments, and for $R=\bigcup_{k=1}^{n} S_{k}$ (with disjoint $S_{k}$ ) in $\mathcal{R}$, we put

$$
\lambda_{R}(A)=\sum_{k=1}^{n} \lambda_{S_{k}}(A)
$$

Now,

$$
\lambda_{T}(A)=\sup _{R \in \mathcal{R}} \lambda_{R}(A)
$$

defines a measure on the $\sigma$-algebra of $T$-measurable sets such that

$$
\int_{[x, y]} f(u) \mathrm{d} \lambda_{T}(u)=\int_{0}^{d(x, y)} f\left(\phi_{x y}(t)\right) \mathrm{d} t
$$

for any $f \in L_{1}(T)$ and $x, y$ in $T$. 
Definition 2.2. Let $T$ be a pointed $\mathbb{R}$-tree also called a rooted real tree, and let $A$ be a closed subset of $T$ containing 0 . We denote by $\mu_{A}$ the positive measure defined by

$$
\mu_{A}=\lambda_{A}+\sum_{a \in A} L(a) \delta_{a}
$$

where $\lambda_{A}$ is the restriction of the length measure on $A, L(a)=\inf _{x \in A \cap[0, a[} d(a, x)$ and $\delta_{a}$ is the Dirac measure on $a$.

This measure takes into account the gaps in $A$ by shifting their mass to the next available point (away from the root). When $L(a)>0$, we say that $a$ is root-isolated in $A$, and we denote by $\hat{A}$ the set of root-isolated points in $A$.

Proposition 2.3. If $T$ is a pointed $\mathbb{R}$-tree and $A$ is a closed subset of $T$ containing $0, L_{\infty}\left(\mu_{A}\right)$ is isometric to the dual space of $L_{1}\left(\mu_{A}\right)$.

When $T$ is separable, this proposition is a direct consequence of a classical theorem.

Proof. We have $L_{1}\left(\mu_{A}\right) \equiv L_{1}\left(\lambda_{A}\right) \oplus_{1} \ell_{1}(\hat{A})$ and $L_{\infty}\left(\mu_{A}\right) \equiv L_{\infty}\left(\lambda_{A}\right) \oplus_{\infty} \ell_{\infty}(\hat{A})$. Hence all we need to prove is $L_{\infty}\left(\lambda_{A}\right) \equiv L_{1}\left(\lambda_{A}\right)^{*}$. We show that the canonical embedding of $L_{\infty}\left(\lambda_{A}\right)$ into $L_{1}\left(\lambda_{A}\right)^{*}$ is onto. Let $\varphi$ be a bounded linear functional on $L_{1}\left(\lambda_{A}\right)$. Then for any $x$ and $y$ in $T, \varphi$ defines by restriction a bounded linear functional on $L_{1}(A \cap[x, y])$; therefore there exists $h_{x y}$ in $L_{\infty}(A \cap[x, y])$ such that

$$
\varphi(f)=\int_{T} f h_{x y} \mathrm{~d} \lambda_{A}
$$

for any $f$ in $L_{1}(A \cap[x, y])$. Any two functions $h_{x y}$ and $h_{x^{\prime} y^{\prime}}$ coincide almost everywhere on $A \cap[x, y] \cap\left[x^{\prime}, y^{\prime}\right]$, and there exists $h$ in $L_{\infty}\left(\lambda_{A}\right)$ such that

$$
\varphi(f)=\int_{T} f h \mathrm{~d} \lambda_{T}
$$

for any $f \in L_{1}\left(\lambda_{A}\right)$ with support included in a finite union of intervals. Such functions form a dense subspace of $L_{1}\left(\lambda_{A}\right)$ and the equality above is therefore valid for any $f$ in $L_{1}\left(\lambda_{A}\right)$.

We define differentiation on a pointed $\mathbb{R}$-tree in the following way.

Definition 2.4. Let $T$ be a pointed $\mathbb{R}$-tree, $A$ a subset of $T$ containing 0 and $f: A \rightarrow \mathbb{R}$. If $a \in A$, we denote by $\hat{a}$ the unique point in $[0, a]$ satisfying $d(a, \hat{a})=L(a)$. When

$$
\lim _{\substack{x \rightarrow \hat{a} \\ x \in[0, a[\cap A}} \frac{f(a)-f(x)}{d(x, a)}
$$

exists, we say that $f$ is differentiable at $a$, and we set $f^{\prime}(a)$ as the value of this limit.

When $A$ is closed, a mapping $f: A \rightarrow \mathbb{R}$ is always differentiable on root-isolated points of $A, f^{\prime}(a)$ being equal in this case to $(f(a)-f(\hat{a})) / L(a)$. When $A=T$, the limit corresponds to the left-derivative of $f_{a}=f \circ \phi_{a}$ at $d(0, a)$, where $\phi_{a}$ is the isometry associated to points 0 and $a$ as in definition 2.1. If $f$ is Lipschitz on $T$, the function $f_{x}=f \circ \phi_{x}$ is differentiable almost everywhere on $[0, d(0, x)]$ for any 
$x$ in $T$; therefore $f$ is differentiable almost everywhere on $T$. Moreover, the change of variable formula (2.1) yields

$$
f(x)-f(0)=\int_{[0, x]} f^{\prime} \mathrm{d} \lambda_{T}
$$

\section{LIPSCHITZ-FREE SPACES OF METRIC TREES}

In this section we compute the Lipschitz-free spaces of a certain class of subsets of $\mathbb{R}$-trees. For this, we integrate bounded measurable functions defined on a closed subset $A$ with respect to the measure $\mu_{A}$ introduced in definition 2.2. We derive the computation of Lipschitz-free spaces of subsets of the real line.

Definition 3.1. A point $t$ of an $\mathbb{R}$-tree $T$ is said to be a branching point of $T$ if $T \backslash\{t\}$ is composed by at least three connected components. We denote by $\operatorname{Br}(T)$ the set of branching points of $T$.

Theorem 3.2. Let $T$ be an $\mathbb{R}$-tree and $A$ be a subset of $T$ such that $\operatorname{Br}(T) \subset \bar{A}$. Then $\mathcal{F}(A)$ is isometric to $L_{1}\left(\mu_{\bar{A}}\right)$.

Proof. As $\mathcal{F}(A)$ is isometric to $\mathcal{F}(\bar{A})$, we may assume that $A$ is closed. We choose a point in $A$ as the origin for $T$ and we prove that $\operatorname{Lip}_{0}(A)$ is isometric to $L_{\infty}\left(\mu_{A}\right)$. For this, we define a linear map $\Phi$ of $L_{\infty}\left(\mu_{A}\right)$ into $\operatorname{Lip}_{0}(A)$ by putting

$$
\Phi(g)(a)=\int_{[0, a]} g \mathrm{~d} \mu_{A} .
$$

The norm of a Lipschitz function $f$ defined on $A$ may be computed on intervals containing 0 . Indeed if $a$ and $b$ are in $A$, there exists $x \in T$ such that $[0, a] \cap[0, b]=$ $[0, x]$ and, as $A$ contains all the branching points of $T$, we know that $x \in A$. One of the quantities $|f(a)-f(x)| / d(x, a)$ or $|f(b)-f(x)| / d(x, b)$ must be greater than or equal to $|f(b)-f(a)| / d(a, b)$ as $x$ belongs to the interval $[a, b]$. We deduce that $\Phi$ is an isometry.

We now prove that $\Phi$ is onto. Let $f$ be in $\operatorname{Lip}_{0}(A)$; we extend $f$ to a Lipschitz mapping $\widetilde{f}$ defined on $T$ and affine on each interval contained in the complement of $A$. The function $\widetilde{f}$ is differentiable almost everywhere on $T$ (in the sense of definition 2.4); therefore $f$ is differentiable $\mu_{A}$-almost everywhere. For any $a \in A$ we have

$$
f(a)=\int_{[0, a]} \widetilde{f}^{\prime} \mathrm{d} \lambda_{T}=\int_{[0, a]} f^{\prime} \mathrm{d} \lambda_{A}+\int_{[0, a] \backslash A} \widetilde{f}^{\prime} \mathrm{d} \lambda_{T} .
$$

The set $B_{a}=[0, a] \backslash A$ is a union of open interval,

$$
\left.B_{a}=\bigcup_{i \in I}\right] \hat{a}_{i}, a_{i}[
$$

so

$$
\int_{B_{a}} \widetilde{f}^{\prime} \mathrm{d} \lambda_{T}=\sum_{i \in I} \frac{f\left(a_{i}\right)-f\left(\hat{a}_{i}\right)}{d\left(\hat{a}_{i}, a_{i}\right)} \times L\left(a_{i}\right)=\sum_{i \in I} L\left(a_{i}\right) f^{\prime}\left(a_{i}\right)=\int_{[0, a]} f^{\prime} \mathrm{d} \nu_{A}
$$

where $\nu_{A}=\sum_{u \in A} L(u) \delta_{u}$.

Hence

$$
f(a)=\int_{[0, a]} f^{\prime} \mathrm{d} \mu_{A}
$$

for any $a$ in $A$ and we get $f=\Phi\left(f^{\prime}\right)$. 
Finally, we know from Proposition 2.3 that $L_{\infty}\left(\mu_{A}\right)$ is isometrically isomorphic to the dual space of $L_{1}\left(\mu_{A}\right)$. The linear isometry $\Phi$ being weak*-weak* continuous, it is the transpose of a linear isometry between $\mathcal{F}(A)$ and $L_{1}\left(\mu_{A}\right)$.

Corollary 3.3. If $T$ is an $\mathbb{R}$-tree, then $\mathcal{F}(T)$ is isometric to $L_{1}(T)$.

The isometry associates to each function $h$ of $L_{1}(T)$ the element $\alpha$ of the Lipschitz-free space defined by $\alpha(f)=\int_{T} f^{\prime} h \mathrm{~d} \lambda_{T}$ i.e. the opposite of the derivative of $h$ in a distribution sense. Conversely, if $\alpha$ is a measure with finite support, the corresponding $L_{1}$-function is defined by $g(t)=\alpha\left(C_{t}\right)$ where $C_{t}=\{x \in T / t \in[0, x]\}$.

For a separable $\mathbb{R}$-tree, we get $\mathcal{F}(T) \equiv L_{1}$. This shows that metric spaces having isometric Lipschitz-free spaces need not be homeomorphic (see 3] for examples of non-Lipschitz isomorphic Banach spaces having linearly isomorphic Lipschitz-free spaces).

Corollary 3.4. Let $T$ be a separable $\mathbb{R}$-tree, and $A$ an infinite subset of $T$ such that $\operatorname{Br}(T) \subset \bar{A}$. If $\bar{A}$ has length measure 0 , then $\mathcal{F}(A)$ is isometric to $\ell_{1}$. If $\bar{A}$ has positive length measure, then $\mathcal{F}(A)$ is isomorphic to $L_{1}$.

Proof. As $T$ is separable, the set of root-isolated points of $\bar{A}$ is at most countable. When $\lambda(\bar{A})>0, \mathcal{F}(A)$ is isometric to one of the spaces $L_{1}, L_{1} \oplus_{1} \ell_{1}^{n}, L_{1} \oplus_{1} \ell_{1}$; the fact that all these spaces are isomorphic to $L_{1}$ results from the Pełczyński decomposition method.

This result allows us to compute the Lipschitz-free space of any subset of $\mathbb{R}$. As an application, we get that infinite discrete subsets of $\mathbb{R}$ have a Lipschitz-free space isometric to $\ell_{1}$. Also, $\mathcal{F}\left(K_{3}\right) \equiv \ell_{1}$ where $K_{3}$ is the usual Cantor set and the Lipschitz-free space of a Cantor set of positive measure is isometric to $L_{1} \oplus_{1} \ell_{1}$; this provides examples of homeomorphic metric spaces having non-isomorphic Lipschitzfree spaces.

Finite subsets of $\mathbb{R}$-trees which contain the branching points usually appear as weighted trees.

Definition 3.5. A weighted tree is a finite connected graph with no cycle, endowed with an edge weighted path metric.

Corollary 3.6. If $T$ is a weighted tree, then $\mathcal{F}(T)$ is isometric to $\ell_{1}^{n}$ where $n=$ $\operatorname{card}(T)-1$.

Embedding a finite metric space into a weighted tree leads to an easy computation of the norm of its Lipschitz-free space (this is not always possible as we shall see in the following section). Let us illustrate this remark with two obvious examples. A metric space $M=\{0,1,2\}$ of cardinality 3 embeds isometrically into a four points weighted tree with edge lengths

$$
\lambda_{0}=\frac{1}{2}\left[d_{01}+d_{02}-d_{12}\right], \lambda_{1}=\frac{1}{2}\left[d_{01}+d_{12}-d_{02}\right], \lambda_{2}=\frac{1}{2}\left[d_{02}+d_{12}-d_{01}\right]
$$

where $d_{i j}$ is the distance between $i$ and $j$. This embedding yields an isometry between $\mathcal{F}(M)$ and a hyperplane of $\ell_{1}^{3}$, and we obtain

$$
\left\|\alpha_{1} \delta_{1}+\alpha_{2} \delta_{2}\right\|=\lambda_{0}\left|\alpha_{1}+\alpha_{2}\right|+\lambda_{1}\left|\alpha_{1}\right|+\lambda_{2}\left|\alpha_{2}\right|
$$


If $M$ is an $(n+1)$-point metric space equipped with the discrete distance $(d(i, j)=1$ if $i \neq j$ ), we obtain in the same way

$$
\left\|\sum_{i=1}^{n} \alpha_{i} \delta_{i}\right\|=\frac{1}{2}\left|\sum_{i=1}^{n} \alpha_{i}\right|+\frac{1}{2} \sum_{i=1}^{n}\left|\alpha_{i}\right| .
$$

\section{Characterization of tree metrics}

In this section we show that subsets of $\mathbb{R}$-trees are the only metric spaces with a Lipschitz-free space isometric to a subspace of an $L_{1}$-space. We use the fact that a metric space isometrically embeds into an $\mathbb{R}$-tree if and only if it is 0-hyperbolic, that is to say satisfies the following property.

Definition 4.1. A metric space is said to satisfy the four-point condition if

$$
d(a, b)+d(c, d) \leq \max (d(a, c)+d(b, d), d(a, d)+d(b, c))
$$

whenever $a, b, c$ and $d$ are in $M$.

Equivalently, the maximum of the three sums $d(a, b)+d(c, d), d(a, c)+d(b, d)$ and $d(a, d)+d(b, c)$ is always attained at least twice. It is fairly straightforward to check that metrics induced by $\mathbb{R}$-trees indeed satisfy this condition. For a proof of the converse, see 4, Chap. 3.

The following theorem relates the aforementioned characterization of $\mathbb{R}$-trees with Lipschitz-free spaces.

Theorem 4.2. Let $M$ be a metric space. The following assertions are equivalent:

(1) $\mathcal{F}(M)$ is isometric to a subspace of an $L_{1}$-space.

(2) $M$ satisfies the four-point condition.

(3) $M$ isometrically embeds into an $\mathbb{R}$-tree.

Proof. (1) $\Rightarrow(2)$ Suppose $M$ is a metric space with a Lipschitz-free space isometric to a subspace of an $L_{1}$-space, and consider four points in $M$ denoted $a_{0}, a_{1}$, $a_{2}$ and $a_{3}$. We will henceforth write $d_{i j}$ in place of $d\left(a_{i}, a_{j}\right)$. The unit ball of $\operatorname{Lip}_{0}\left(\left\{a_{0}, a_{1}, a_{2}, a_{3}\right\}\right)$ is isometric to the convex set of $\mathbb{R}^{3}$ defined by the conditions

$$
|x| \leq d_{01} \quad ;|y| \leq d_{02} \quad ;|z| \leq d_{03} \quad ;|y-x| \leq d_{12} \quad ; \quad|z-x| \leq d_{13} \quad ;|z-y| \leq d_{23} .
$$

Since $\mathcal{F}\left(\left\{a_{0}, a_{1}, a_{2}, a_{3}\right\}\right)$ is isometric to a subspace of $L_{1}$, this polytope is the projection of a cube and therefore all its faces admit a center of symmetry. The face obtained when making the third inequality to bind is characterized by

$$
\begin{aligned}
d_{03}-d_{13} \leq x \leq d_{01} \\
d_{03}-d_{23} \leq y \leq d_{02} \\
x-d_{12} \leq y \leq x+d_{12}
\end{aligned}
$$

in the affine plane of equation $z=d_{03}$.

For any real numbers $a, b, c, d, e, f$, the planar convex set defined by

$$
\begin{aligned}
a & \leq x \leq b \\
c & \leq y \leq d \\
x+e & \leq y \leq x+f
\end{aligned}
$$


is either empty or centrally symmetric if and only if one of the following nine conditions holds:

$$
\begin{array}{ccc}
b \leq a & d \leq c & f \leq e \\
d \leq a+e & b+f \leq c & a+b+e+f=c+d \\
\left\{\begin{aligned}
d & \leq a+f \\
b+e & \leq c
\end{aligned}\right. & \left\{\begin{aligned}
& b+f \leq d \\
& c \leq \leq a+e
\end{aligned}\right. & \left\{\begin{aligned}
a+f \leq c \\
d \leq b+e
\end{aligned}\right.
\end{array}
$$

Those different possibilities encompass segments, rectangles, parallelograms and symmetric hexagons. Applying this to $a=d_{03}-d_{13}, b=d_{01}, c=d_{03}-d_{23}$, $d=d_{02}, e=-d_{12}$ and $f=d_{12}$ yields that one of the following conditions holds:

$$
\begin{aligned}
& d_{03}=d_{01}+d_{13} \\
& d_{03}=d_{02}+d_{23} \\
& \left\{\begin{array}{l}
d_{01}+d_{23} \leq d_{03}+d_{12} \\
d_{02}+d_{13} \leq d_{03}+d_{12}
\end{array}\right. \\
& \left\{\begin{array}{lll}
d_{02} & = & d_{01}+d_{12} \\
d_{23} & = & d_{12}+d_{13}
\end{array}\right. \\
& \left\{\begin{array}{l}
d_{01}=d_{02}+d_{12} \\
d_{13}=d_{12}+d_{23}
\end{array}\right. \\
& d_{01}+d_{23}=d_{02}+d_{13}
\end{aligned}
$$

Both (4.1) and (4.2) imply (4.3). Also, each of (4.4) and (4.5) imply (4.6), which leaves us with

$$
\begin{gathered}
\left\{\begin{array}{l}
d_{01}+d_{23} \leq d_{03}+d_{12} \\
d_{02}+d_{13} \leq d_{03}+d_{12}
\end{array}\right. \\
d_{01}+d_{23}=d_{02}+d_{13},
\end{gathered}
$$

either condition implying $d_{01}+d_{23} \leq \max \left(d_{02}+d_{13}, d_{03}+d_{12}\right)$.

$(2) \Rightarrow(3)$ see 4 .

$(3) \Rightarrow(1)$ results from Corollary 3.3 .

In the special case when $M$ is separable, the $L_{1}$-space in condition (1) can be taken as $L_{1}$, any separable isometric subspace of an $L_{1}$-space being an isometric subspace of $L_{1}$. In case $M$ is finite, it can be replaced by $\ell_{1}^{N}$ where $N=2 \times$ $\operatorname{Card}(M)-3$ (it is shown in 2] that a finite metric space with the four-point property isometrically embeds into a weighted tree with at most $2 \times \operatorname{Card}(M)-2$ vertices).

Note that a polyhedral space isometrically embeds into $L_{1}$ if and only if its 3-dimensional subspaces do so (the so-called Hlawka's inequality which characterizes this property involves only three vectors). As far as Lipschitz-free spaces 
$\mathcal{F}(M)$ are concerned, embeddability of the 3-dimensional subspaces corresponding to Lipschitz-free spaces of four-point subsets of $M$ is enough, and we obtain a canonical embedding.

\section{LIPSCHITZ-FREE SPACES ISOMORPHIC TO A SUBSPACE OF $L_{1}$}

We show in this section a very natural gluing result: if $M$ is a metric space of finite radius composed of uniformly apart subsets $M_{\gamma}, \mathcal{F}(M)$ is essentially the $\ell_{1}$-sum of the Lipschitz-free spaces $\mathcal{F}\left(M_{\gamma}\right)$.

Proposition 5.1. Let $\Gamma$ be a pointed set (the distinguished point being denoted 0 ) and $M=\bigcup_{\gamma \in \Gamma} M_{\gamma}$ a metric space. If there exist positive constants $\alpha$ and $\beta$ such that $\alpha \leq d(x, y) \leq \beta$ whenever $x$ and $y$ belong to distinct $M_{\gamma}$ 's, then we have the isomorphism

where $\Gamma^{*}=\Gamma \backslash\{0\}$.

$$
\mathcal{F}(M) \simeq\left(\sum_{\gamma \in \Gamma} \mathcal{F}\left(M_{\gamma}\right)\right)_{\ell_{1}} \oplus_{1} \ell_{1}\left(\Gamma^{*}\right)
$$

The $\ell_{1}$-space compensates for the loss of dimensions due to the choice of a base point in each $M_{\gamma}$ : each connected component of $M \backslash M_{0}$ contributes for one dimension in this $\ell_{1}$-space.

Proof. For each $\gamma \in \Gamma$, we choose a base point $a_{\gamma}$ in $M_{\gamma}$ in order to define $\mathcal{F}\left(M_{\gamma}\right)$, and use $a_{0}$ to define $\mathcal{F}(M)$. We consider the following two maps:

$$
\Phi:\left\{\begin{array}{cl}
\left(\sum_{\gamma \in \Gamma} \operatorname{Lip}_{0}\left(M_{\gamma}\right)\right)_{\ell_{\infty}} \oplus_{\infty} \ell_{\infty}\left(\Gamma^{*}\right) & \rightarrow \operatorname{Lip}_{0}(M) \\
\left(\left(f_{\gamma}\right)_{\gamma \in \Gamma},\left(\lambda_{\gamma}\right)_{\gamma \in \Gamma^{*}}\right) & \mapsto f=\left\{\begin{array}{cc}
f_{0} & \text { on } M_{0} \\
f_{\gamma}+\lambda_{\gamma} & \text { on } M_{\gamma}
\end{array}\right.
\end{array}\right.
$$

and

$$
\Psi:\left\{\begin{array}{cl}
\operatorname{Lip}_{0}(M) & \rightarrow\left(\sum_{\gamma \in \Gamma} \operatorname{Lip}_{0}\left(M_{\gamma}\right)\right)_{\ell_{\infty}} \oplus_{\infty} \ell_{\infty}\left(\Gamma^{*}\right) \\
f & \mapsto\left(\left(\left.f\right|_{M_{\gamma}}-f\left(a_{\gamma}\right)\right)_{\gamma \in \Gamma},\left(f\left(a_{\gamma}\right)\right)_{\gamma \in \Gamma^{*}}\right)
\end{array}\right.
$$

First of all let us show that $\Phi$ is well-defined and has norm less than $2(\alpha+\beta+1) / \alpha$. If $u=\left(\left(f_{\gamma}\right)_{\gamma \in \Gamma},\left(\lambda_{\gamma}\right)_{\gamma \in \Gamma^{*}}\right)$, we have for any $x$ in $M_{\sigma}$ and $y$ in $M_{\tau}$,

$$
|\Phi(u)(y)-\Phi(u)(x)| \leq\left|f_{\sigma}(x)\right|+\left|f_{\tau}(y)\right|+\left|\lambda_{\sigma}\right|+\left|\lambda_{\tau}\right|
$$

(with $\lambda_{0}=0$ ). Now, assuming $\sigma \neq \tau$, we get

$$
\left|f_{\sigma}(x)\right| \leq\left\|f_{\sigma}\right\| d\left(x, a_{\sigma}\right) \leq\left\|f_{\sigma}\right\|\left(d(x, y)+d\left(y, a_{\sigma}\right)\right) \leq\|u\|(d(x, y)+\beta) .
$$

The inequality $1 \leq \frac{1}{\alpha} d(x, y)$ yields

$$
\left|f_{\sigma}(x)\right| \leq\left(1+\frac{\beta}{\alpha}\right)\|u\| d(x, y) \quad \text { and } \quad\left|\lambda_{\sigma}\right| \leq \frac{1}{\alpha}\|u\| d(x, y),
$$

these upper bounds being also valid for $\tau$. Hence

$$
|\Phi(u)(y)-\Phi(u)(x)| \leq 2 \frac{\alpha+\beta+1}{\alpha}\|u\| d(x, y) .
$$


The latter inequality holds if $x$ and $y$ belong to the same $M_{\sigma}$, and we conclude that $\Phi(u)$ is a Lipschitz map of norm less than $2 \frac{\alpha+\beta+1}{\alpha}\|u\|$. The map $\Psi$ is the inverse of $\Phi$, and we have $\|\Psi\| \leq \max (1, \beta)$. The isomorphism $\Phi$ being weak* weak* continuous, it is the transpose of a linear isomorphism between $\mathcal{F}(M)$ and $\left(\oplus_{\ell_{1}} \mathcal{F}\left(M_{\gamma}\right)\right) \oplus_{1} \ell_{1}\left(\Gamma^{*}\right)$.

Combined with the results of the preceding section, this gives canonical examples of Lipschitz-free spaces isomorphic to a subspace of $L_{1}$ (and therefore a unique isometric predual by [7]).

Examples of spaces $\mathcal{F}(M)$ which are unique isometric preduals are plentiful in 9]. Kalton showed the following result: if $M$ is an arbitrary metric space and $\varepsilon>0$, then the space $\mathcal{F}(M)$ is $(1+\varepsilon)$-isometric to a subspace of $\left(\sum_{k \in \mathbb{Z}} \mathcal{F}\left(M_{k}\right)\right)_{\ell_{1}}$ where $M_{k}=\left\{x \in M / d(x, 0) \leq 2^{k}\right\}$. It is then deduced that when $M$ is uniformly discrete (i.e. $\left.\inf _{x \neq y} d(x, y)>0\right), \mathcal{F}(M)$ is a Schur space with the Radon-Nikodým property and the approximation property. In this case, $\mathcal{F}(M)$ is a unique isometric predual since it has the Radon-Nikodým property (see [5] p. 144). Also, according to Bonic, Frampton and Tromba ([1], corrected in [12]), if $K$ is a compact subset of a finite-dimensional normed space and $0<\alpha<1$, the metric space $K$ equipped with the distance $d(x, y)=\|y-x\|^{\alpha}$ has a Lipschitz-free space $\left(\operatorname{denoted} \mathcal{F}_{\alpha}(K)\right)$ isomorphic to $\ell_{1}$. Hence $\mathcal{F}_{\alpha}(A)$ is a unique isometric predual whenever $A$ is a subset of a finite-dimensional normed space. Note that in [9] Kalton showed that if $K$ is

a compact convex subset of $\ell_{2}$ and is infinite-dimensional, then $\mathcal{F}_{\alpha}(K)$ cannot be isomorphic to $\ell_{1}$.

\section{ACKNowledgments}

The author thanks Guillaume Aubrun for having introduced him to the theory of zonoids and Gilles Lancien for useful discussions and comments.

\section{REFERENCES}

1. R. Bonic, J. Frampton, and A. Tromba, $\Lambda$-manifolds, J. Functional Analysis 3 (1969), 310320. MR0238359(38:6635)

2. P. Buneman, A note on the metric properties of trees, J. Combinatorial Theory Ser. B 17 (1974), 48-50. MR0363963 (51:218)

3. Y. Dutrieux and V. Ferenczi, The Lipschitz free Banach spaces of $C(K)$-spaces, Proc. Amer. Math. Soc. 134 (2006), no. 4, 1039-1044. MR2196036 (2006h:46003)

4. S.N. Evans, Probability and real trees, Lecture Notes in Mathematics, vol. 1920, Springer, Berlin, 2008. MR 2351587

5. G. Godefroy, Existence and uniqueness of isometric preduals: a survey, Banach space theory, Contemp. Math., vol. 85, Amer. Math. Soc., Providence, RI, 1989, pp. 131-193. MR.983385 (90b:46035)

6. G. Godefroy and N.J. Kalton, Lipschitz-free Banach spaces, Studia Math. 159 (2003), no. 1, 121-141. MR2030906 (2004m:46027)

7. G. Godefroy and M. Talagrand, Classes d'espaces de Banach à prédual unique, C. R. Acad. Sci. Paris Sér. I Math. 292 (1981), no. 5, 323-325. MR608845 (82b:46020)

8. P. Goodey and W. Weil, Zonoids and generalisations, Handbook of convex geometry, Vols. A, B, North-Holland, Amsterdam, 1993, pp. 1297-1326. MR1243010 (95g:52015)

9. N.J. Kalton, Spaces of Lipschitz and Hölder functions and their applications, Collect. Math. 55 (2004), no. 2, 171-217. MR2068975 (2005c:46113)

10. A. Naor and G. Schechtman, Planar earthmover is not in $L_{1}$, SIAM J. Comput. 37 (2007), no. 3, 804-826. MR2341917 
11. W. Rudin, Real and complex analysis, third ed., McGraw-Hill Book Co., New York, 1987. MR 924157 (88k:00002)

12. N. Weaver, Lipschitz algebras, World Scientific Publishing Co. Inc., River Edge, NJ, 1999. MR.1832645 (2002g:46002)

Institut de Mathématiques de Jussieu - Projet Analyse Fonctionnelle, Université Paris 6, Boîte 186, 4 Place Jussieu, 75252 Paris Cédex 05, France

E-mail address: godard@math.jussieu.fr 